$\mathrm{ULB} / 229 / \mathrm{CQ} / 97 / 2$

\title{
Generalized Morse potential: symmetry and satellite potentials
}

\author{
A. Del Sol Mesa ${ }^{a, *}$, C. Quesne ${ }^{b, \dagger}$, Yu. F. Smirnov ${ }^{c, \ddagger}$ \\ ${ }^{a}$ Instituto de Física, UNAM, Apdo Postal 20-364, 01000 México DF, Mexico \\ ${ }^{b}$ Physique Nucléaire Théorique et Physique Mathématique, Université Libre de Bruxelles, \\ Campus de la Plaine CP229, Boulevard du Triomphe, B-1050 Brussels, Belgium \\ ${ }^{c}$ Instituto de Ciencias Nucleares, UNAM, Apdo Postal 70-543, 04510 México DF, Mexico
}

\begin{abstract}
We study in detail the bound state spectrum of the generalized Morse potential (GMP), which was proposed by Deng and Fan as a potential function for diatomic molecules. By connecting the corresponding Schrödinger equation with the Laplace equation on the hyperboloid and the Schrödinger equation for the Pöschl-Teller potential, we explain the exact solvability of the problem by an $s o(2,2)$ symmetry algebra, and obtain an explicit realization of the latter as $s u(1,1) \oplus s u(1,1)$. We prove that some of the $s o(2,2)$ generators connect among themselves wave functions belonging to different GMP's (called satellite potentials). The conserved quantity is some combination of the potential parameters instead of the level energy, as for potential algebras. Hence, so $(2,2)$ belongs to a new class of symmetry algebras. We also stress the usefulness of our algebraic results for simplifying the calculation of Frank-Condon factors for electromagnetic transitions between rovibrational levels based on different electronic states.
\end{abstract}

Short title: Generalized Morse potential

PACS: 03.65.Fd, 33.10.Gx, 33.10.Cs

To be published in J. Phys. A

*E-mail: antonio@sysul1.ifisicacu.unam.mx

${ }^{\dagger}$ Directeur de recherches FNRS; E-mail: cquesne@ulb.ac.be

${ }^{\ddagger}$ E-mail: smirnov@xochitl.nuclecu.unam.mx 


\section{Introduction}

It is well known that the factorization method of Infeld and Hull [1], the algebraic approach of Alhassid et al [2, 3, 4, 5], and/or the SUSYQM superalgebraic scheme for shape-invariant potentials [6] allow one to connect with one another wave functions $\Psi^{(m)}$ corresponding to a set of potentials $V^{(m)}, m=0,1,2, \ldots$ The wave functions $\Psi^{(m)}$ satisfy the Schrödinger equations

$$
\left[-\frac{\hbar^{2}}{2 \mu} \frac{d^{2}}{d x^{2}}+V^{(m)}(x)\right] \Psi^{(m)}(x)=E \Psi^{(m)}(x)
$$

with the same energy eigenvalue $E$. In the factorization method, the connections between these functions are given by the relations

$$
H^{( \pm)} \Psi^{(m)}(x)=\Psi^{(m \pm 1)}(x)
$$

where $H^{( \pm)}$are $m$-dependent, first-order differential operators. Explicit expressions for the ladder operators $H^{( \pm)}$were found in [1] for various types of potentials.

In the algebraic approach [2, 3, 4, 5], the $m$-dependence of the ladder operators is eliminated by introducing some auxiliary variables, so that the resulting operators coincide with the generators of some algebra $G(G=s u(2), s o(2,1), s o(2,2)$, etc). A definite irreducible representation (irrep) $D^{w}$ of $G$ is realized in the space of wave functions $\Psi^{(m)}$. The number of interconnected functions $\Psi^{(m)}$ (and corresponding potentials $V^{(m)}$ ) can be finite $\left(m \leq n-1\right.$, where $n$ is the dimension of the irrep $D^{w}$ of the compact algebra $G$ ) or infinite ( $D^{w}$ belongs to the discrete series of unitary irreps of the noncompact algebra $G$ ).

SUSYQM provides an alternative algebraization of the factorization method, wherein the ladder operators give rise to supercharge operators $Q^{ \pm}[6,7]$. Together with the supersymmetric Hamiltonian, the latter generate an su(1/1) superalgebra.

A common feature of the various approaches [1, 2, 3, 4, 5, 6, 7] is the fact that taking some initial potential $V^{(i)}$, one can construct a set of potentials $V^{(0)}, V^{(1)}, \ldots, V^{(i-1)}$, $V^{(i+1)}, \ldots$, associated with it. If $V^{(i)}$ has a finite number of levels (bound states) $E_{s}^{(i)}$, $s=0,1, \ldots, l$, then the potential $V^{(m)}(m<i)$ has the same levels $E_{s}^{(i)}$ except for $i-m$ of them, i.e., $s=0,1, \ldots, l-i+m$. The corresponding wave functions of such potentials, 
which may be called satellite potentials, are connected by eq. (1.2). In [1, 2, 3, 4, 5, 6, 7], it was assumed that the level energy $E$ is constant (in [2, 3, 4, 5], $E$ is unambigously connected with the irrep signature $w)$. Applying the algebra $G$ generators $H^{( \pm)}$on a state belonging to a potential of this set gives rise to a state belonging to another satellite potential. Therefore the algebra $G$ was called the potential algebra [2], or the algebra describing the fixed energy states of a family of systems with quantized coupling constants [5].

In the present paper, we consider a potential suggested by Deng and Fan in 1957 [8] to describe diatomic molecular energy spectra and electromagnetic transitions, and referred to as the generalized Morse potential (GMP). As the standard one [9],

$$
V(x)=D\left[1-e^{-a\left(x-x_{e}\right)}\right]^{2}
$$

where $D, a$, and $x_{e}$ are some constants, it has the advantage of automatically including some anharmonicity effects, while admitting an exact analytical solution, allowing one to easily calculate various molecular characteristics. In contrast, it has none of the three well-known limitations of the Morse potential, namely (i) to be defined in the interval $-\infty<x<\infty$, including the non-physical region $x<0$, (ii) to be finite at $x=0$ unlike the true internuclear interaction, and (iii) to contain only two terms in the Dunham expansion for the energies $E_{n}$ with respect to $(n+1 / 2)$,

$$
E_{n}=\sqrt{\frac{2 D}{\mu}} a \hbar(n+1 / 2)-\frac{a^{2} \hbar^{2}}{2 \mu}(n+1 / 2)^{2}
$$

whereas higher order terms are needed for a good description of the vibrational levels of some diatomic molecules [8]. The GMP therefore provides an interesting alternative to some recent attempts to eliminate the Morse potential defects, based on computer calculations [10].

Our aim is to explain the exact solvability of the GMP by determining its symmetry algebra. For such a purpose, it will prove convenient to relate the Schrödinger equation for the GMP to other equations with known symmetry algebras, namely the Laplace equation on the hyperboloid [11], and the Schrödinger equation for the Pöschl-Teller potential (PTP) [5]. 
The GMP symmetry algebra, resulting from such connections, will turn out to be distinct from the potential algebras, and to belong to a new class of symmetry algebras, wherein the operators (generators) $H^{( \pm)}$connect with one another wave functions $\Psi^{(m)}(x)$ satisfying the equations

$$
\left[-\frac{\hbar^{2}}{2 \mu} \frac{d^{2}}{d x^{2}}+V^{(m)}(x)\right] \Psi^{(m)}(x)=E^{(m)} \Psi^{(m)}(x)
$$

with $m$-dependent eigenvalues $E^{(m)}$. Contrary to the case of eq. (1.1), the conserved (constant) quantity for the set of satellite potentials $V^{(0)}, V^{(1)}, \ldots, V^{(m)}, \ldots$ will not be the level energy $E$, but some combination $f\left(a_{m}\right)$ of the potential parameters $a_{m}$ :

$$
f\left(a_{m}\right)=\text { constant }
$$

One may still consider some first-order differential operators $H^{( \pm)}$connecting the wave functions of neighbouring satellite potentials, and satisfying eq. (1.2). They are similar to those of the factorization method [1], except for the fact that they do not conserve the energy eigenvalue $E$. Under certain conditions, which are fulfilled for the GMP, they may generate some algebra $G$, associated with the satellite potentials. In this algebraic case, the wave functions $\Psi^{(m)}(x), m=0,1, \ldots$, form a basis of an irrep $D^{w}$ of the algebra $G$ and its signature $w$ is connected with the constant $f\left(a_{m}\right)$ of eq. (1.6). Such an approach therefore allows one to construct a set of satellite potentials $V^{(m)}(m=0,1,2, \ldots)$, which are different in the general case from potentials produced by the factorization method [1], by potential algebras [2, 3, 4, 5], or by SUSYQM [6, 7].

This paper is organized as follows. In section 2, the GMP is reviewed, and its bound state spectrum and corresponding normalized wave functions are given. Its connections with the Laplace operator on the hyperboloid and with the Pöschl-Teller potential (PTP) are established in section 3 , and used in section 4 to construct a symmetry algebra $s o(2,2)$ related to the new type of satellite potentials. Some concluding remarks are given in section 5 . 


\section{The generalized Morse potential}

The GMP, introduced by Deng and Fan [8], and related to the Manning-Rosen potential [1, 12] (also called Eckart potential by some authors [7]), is defined by

$$
V(r)=D\left[1-\frac{b}{e^{a r}-1}\right]^{2} \quad b=e^{a r_{e}}-1
$$

where $0 \leq r<\infty$, and $D, b, a$ are some parameters regulating the depth, the position of the minimum $r_{e}$, and the radius of the potential. Notice that it is defined on the same range, and has the same behaviour for $r \rightarrow 0$, as the true internuclear potential in diatomic molecules.

In the dimensionless variable $x=\operatorname{ar}(0 \leq x<\infty)$, the Schrödinger equation for the GMP can be written as

$$
\left(-\frac{d^{2}}{d x^{2}}+v(x)\right) \psi(x)=\epsilon \psi(x) \quad v(x) \equiv k\left(1-\frac{b}{e^{x}-1}\right)^{2}
$$

where $\Psi(r)=\sqrt{a} \psi(x)$, and

$$
k \equiv \frac{2 \mu D}{a^{2} \hbar^{2}} \quad \epsilon \equiv \frac{2 \mu E}{a^{2} \hbar^{2}} .
$$

As was shown in [8], it is solvable in an analytical way. The introduction of a new variable $y$ and a new function $F(y)$, defined by

$$
\begin{aligned}
y & =\left(e^{x}-1\right)^{-1} \\
\psi(x) & =\Phi(y)=\frac{y^{\alpha}}{(1+y)^{\beta}} F(y)
\end{aligned}
$$

respectively, indeed transforms eq. (2.2) into the following equation

$$
y(1+y) \frac{d^{2} F}{d y^{2}}+[2(\alpha-\beta+1) y+(2 \alpha+1)] \frac{d F}{d y}+\left[(\alpha-\beta)^{2}+(\alpha-\beta)+C\right] F(y)=0
$$

equivalent to the hypergeometric one, provided $\alpha, \beta$, and $C$ are chosen so as to satisfy the relations

$$
\begin{aligned}
\alpha & =\sqrt{k-\epsilon}=\sqrt{\frac{2 \mu(D-E)}{a^{2} \hbar^{2}}} \\
\beta & =\sqrt{\alpha^{2}+k b(b+2)}=\sqrt{k(b+1)^{2}-\epsilon}=\sqrt{\frac{2 \mu}{a^{2} \hbar^{2}}\left[D(b+1)^{2}-E\right]} \\
C & =-k b^{2}=-\frac{2 \mu}{a^{2} \hbar^{2}} b^{2} D .
\end{aligned}
$$


A solution of (2.6) is $F(y)={ }_{2} F_{1}(d, e ; 2 \alpha+1 ;-y)$, where

$$
d=\alpha-\beta+l \quad e=\alpha-\beta+1-l \quad l \equiv \frac{1}{2}(1+\sqrt{1-4 C})=\frac{1}{2}\left(1+\sqrt{1+4 k b^{2}}\right)
$$

Bound states correspond to those functions $F(y)$ that reduce to polynomials, i.e., for which $d=-n$, where $n$ is some nonnegative integer. From eqs. (2.8) and (2.10), it is then clear that $\alpha$ and $\beta$ are $n$-dependent, and solutions of the equations

$$
\begin{aligned}
& \beta_{n}-\alpha_{n}=n+l \\
& \beta_{n}^{2}-\alpha_{n}^{2}=k b(b+2) .
\end{aligned}
$$

One finds

$$
\alpha_{n}=\frac{1}{2}\left(\frac{k b(b+2)}{n+l}-n-l\right) \quad \beta_{n}=\frac{1}{2}\left(\frac{k b(b+2)}{n+l}+n+l\right) .
$$

From eq. (2.7), it follows that $\epsilon_{n}$ can be expressed in terms of $\alpha_{n}$ as

$$
\epsilon_{n}=k-\alpha_{n}^{2}
$$

So, we conclude that the energy eigenvalues are given by

$$
E_{n}=D-\frac{a^{2} \hbar^{2}}{8 \mu}\left(n+l-\frac{b(b+2) k}{n+l}\right)^{2}
$$

and that the corresponding eigenfunctions are

$$
\Psi_{n}(r)=N_{n} y^{\alpha_{n}}(1+y)^{-\beta_{n}} F_{1}\left(-n,-n+1-2 l ; 2 \alpha_{n}+1 ;-y\right) \quad y=\left(e^{a r}-1\right)^{-1}
$$

where $N_{n}$ is some normalization coefficient, determined by the condition

$$
\int_{0}^{\infty} d r\left|\Psi_{n}(r)\right|^{2}=\int_{0}^{\infty} d x\left|\psi_{n}(x)\right|^{2}=\int_{0}^{\infty} d y[y(1+y)]^{-1}\left|\Phi_{n}(y)\right|^{2}=1
$$

In eqs. (2.15) and (2.16), the quantum number $n$ takes a finite set of values

$$
n=0,1,2, \ldots, n_{\max } \quad n_{\max } \equiv \sqrt{k b(b+2)}-l
$$

where the quantity defining $n_{\max }$ is assumed integer (otherwise one has to take its integer part). 
It is not a trivial matter to derive a closed expression for the normalization coefficient $N_{n}$ of the eigenfunctions (2.16), which was not given in [8]. As shown in the appendix, this can most easily be done by using SUSYQM techniques. The result can be rewritten as

$$
N_{n}=\left(\frac{a\left(\alpha_{n}+n+l\right) \Gamma\left(2 \alpha_{n}+n+1\right) \Gamma\left(2 \alpha_{n}+n+2 l\right)}{n !(n+l) \Gamma\left(2 \alpha_{n}\right) \Gamma\left(2 \alpha_{n}+1\right) \Gamma(n+2 l)}\right)^{1 / 2}
$$

Making an expansion of (2.15) in terms of powers of $(n+1 / 2)$, we get [8]

$$
E_{n}=\epsilon(0)+\epsilon(1)(n+1 / 2)-\epsilon(2)(n+1 / 2)^{2}+\epsilon(3)(n+1 / 2)^{3}-\cdots
$$

where $\epsilon(0), \epsilon(1), \epsilon(2)$, and $\epsilon(3)$ are coefficients depending on the parameters of the potential function. This means that the GMP includes terms of arbitrary order in the Dunham expansion. It is easy to verify that all corrections $\epsilon(k) \rightarrow 0(k \geq 3)$ whenever $b \rightarrow \infty$. Therefore, in this limit, the eigenvalues (2.15) coincide with those of the Morse potential, given in eq. (1.4).

On the other hand, using the well-known limit relation between Gauss and confluent hypergeometric functions ${ }_{2} F_{1}(a, b, c ; z / b) \rightarrow{ }_{1} F_{1}(a, c ; z)$, when $b \rightarrow \infty\left(\right.$ or $\left.r_{e} \gg 1\right)$ [13, and considering the region $r \gg 1$, we obtain that the GMP eigenfunctions (2.16) can be reduced to those of the Morse potential.

The interrelation between the Morse potential and the GMP is illustrated in Fig.1. It is clear that for rather large values of $r_{e}(a=1)$, these potentials are very close to each other in the regions $r \sim r_{e}$ and $r>r_{e}$. However, they are very different at $r \sim 0$. If both potentials are rather deep $(D \gg 1)$, they could be well approximated, in the region $r \sim r_{e}$, by a harmonic oscillator potential with frequency $\hbar \omega=\epsilon(1)$ (see eq. (2.20)).

Usually, the existence of an exact analytical solution of the Schrödinger equation for some system can be explained by the fact that the corresponding Hamiltonian has some symmetry algebra. In the next two sections, we shall proceed to determine the latter for the GMP.

\section{Connections with the Laplace operator on the hy- perboloid and the Pöschl-Teller potential}

As well known, in order to find the symmetry algebra related to a one-dimensional Schrödinger equation, it is useful to map the latter into a problem in a higher-dimensional 
space. For instance, by using some transformation, Alhassid et al [2] were able to map the Schrödinger equation for the Morse potential into a two-dimensional harmonic oscillator.

Following this line of thought, we will embed our one-dimensional GMP problem into a three-dimensional space. Namely, we will show below that the Schrödinger equation for the GMP can be connected with the Laplace-Beltrami-Casimir one on the four-dimensional hyperboloid defined by

$$
x_{1}^{2}+x_{2}^{2}-x_{3}^{2}-x_{4}^{2}=\rho^{2}>0
$$

whose symmetry algebra is $s o(2,2)$.

Using the Casimir operator of this algebra, we will derive the discrete spectrum of the GMP, and show that the corresponding wave functions can be connected with the eigenfunctions of the Laplace operator on the hyperboloid, or, equivalently, with those of the Schrödinger equation for the Pöschl-Teller potential (PTP),

$$
V_{P T}=\frac{\lambda^{2}}{\sinh ^{2} \theta}-\frac{\bar{\lambda}^{2}}{\cosh ^{2} \theta} \quad 0 \leq \theta<\infty .
$$

We will therefore conclude that the exact solvability of the Schrödinger equation for the GMP is explained by its connection with the $s o(2,2)$ algebra.

Let us analyze the symmetry problem for the GMP in detail. We start by considering the four-dimensional Minkowski space determined by the relations [11, 14]

$$
\begin{array}{ll}
x_{1}=\rho \cosh \theta \cos \varphi & x_{2}=\rho \cosh \theta \sin \varphi \\
x_{3}=\rho \sinh \theta \cos \phi & x_{4}=\rho \sinh \theta \sin \phi
\end{array}
$$

where $0 \leq \theta<\infty, 0 \leq \varphi<2 \pi, 0 \leq \phi<2 \pi, \rho \geq 0$. In such coordinates, the LaplaceBeltrami-Casimir operator takes the form

$$
\Delta_{\Omega}=-\frac{1}{\sinh \theta \cosh \theta} \frac{\partial}{\partial \theta}\left(\cosh \theta \sinh \theta \frac{\partial}{\partial \theta}\right)+\frac{1}{\cosh ^{2} \theta} \frac{\partial^{2}}{\partial \varphi^{2}}-\frac{1}{\sinh ^{2} \theta} \frac{\partial^{2}}{\partial \phi^{2}} .
$$

Its eigenvalues can be written as

$$
\lambda=-L(L+2) \quad L=0,1,2, \ldots, L_{\max }
$$


and its eigenfunctions $\Psi_{L m_{1} m_{2}}(\theta, \varphi, \phi)$ can be factorized as follows

$$
\Psi_{L m_{1} m_{2}}(\theta, \varphi, \phi)=e^{i m_{1} \varphi} \psi_{L m_{1} m_{2}}(\theta) e^{i m_{2} \phi}
$$

where $\psi_{L m_{1} m_{2}}(\theta)$ satisfies the equation

$$
\begin{array}{r}
{\left[-\frac{\partial^{2}}{\partial \theta^{2}}-(\tanh \theta+\operatorname{coth} \theta) \frac{\partial}{\partial \theta}-\frac{m_{1}^{2}}{\cosh ^{2} \theta}+\frac{m_{2}^{2}}{\sinh ^{2} \theta}\right] \psi_{L m_{1} m_{2}}(\theta)} \\
=-L(L+2) \psi_{L m_{1} m_{2}}(\theta) .
\end{array}
$$

We notice that according to the choice of coordinates (3.3), the problem of determining the eigenvalues and eigenfunctions of the Laplace-Beltrami-Casimir operator corresponds to the reduction

$$
s o(2,2) \supset s o_{1}(2) \oplus s o_{2}(2)
$$

of the $s o(2,2)$ algebra into its subalgebras $s o_{1}(2)$ and $s o_{2}(2)$, generated by the operators $-i \partial / \partial \varphi$ and $-i \partial / \partial \phi$, respectively. The eigenvalues

$$
m_{1}, m_{2}=0, \pm 1, \pm 2, \ldots
$$

of the latter enumerate the irreps of these subalgebras. The quantum number

$$
L=0,1,2, \ldots
$$

characterizes the irrep of the algebra $s o(2,2)$, belonging to the discrete series of its most degenerate unitary irreps [14].

It is well known from the general theory of $s o(p, q)$ irreps [14, that in a given irrep $D^{L}$ of $s o(2,2)$, the admissible values of the quantum numbers $m_{1}$ and $m_{2}$ are given by the condition

$$
\left|m_{1}\right|-\left|m_{2}\right|=L+2+2 n \quad n=0,1,2, \ldots
$$

Therefore, if $m_{1}$ and $m_{2}$ are fixed in eq. (3.7), then

$$
L=L_{\max }, L_{\max }-2, \ldots, 1 \text { or } 0
$$

depending on

$$
L_{\text {max }}=\left|m_{1}\right|-\left|m_{2}\right|-2
$$


being odd or even, respectively. Thus, the total number of possible $L$ values is

$$
\nu_{\max }=\frac{1}{2}\left(\left|m_{1}\right|-\left|m_{2}\right|\right) \quad \text { or } \quad \frac{1}{2}\left(\left|m_{1}\right|-\left|m_{2}\right|-1\right)
$$

for $L_{\max }$ even or odd, respectively.

Before bringing the discussion further, it is interesting to make the following substitution

$$
\psi_{L_{m_{1} m_{2}}}(\theta)=\cosh ^{-1 / 2} \theta \sinh ^{-1 / 2} \theta \chi(\theta)
$$

transforming the Laplace equation (3.7) into the Schrödinger equation for the PTP

$$
\left[-\frac{d^{2}}{d \theta^{2}}+\frac{\left(m_{2}^{2}-1 / 4\right)}{\sinh ^{2} \theta}-\frac{\left(m_{1}^{2}-1 / 4\right)}{\cosh ^{2} \theta}\right] \chi(\theta)=\bar{\epsilon} \chi(\theta)
$$

where

$$
\bar{\epsilon}=-(L+1)^{2}=-\left(\left|m_{1}\right|-\left|m_{2}\right|-1-2 n\right)^{2} .
$$

Equation (3.14) shows the number of bound states in the PTP for fixed amplitudes $m_{1}$ and $m_{2}$ of its attractive and repulsive parts. If

$$
\left|m_{2}\right|-\left|m_{1}\right| \geq-1
$$

then there are no bound states.

Let us now establish the connection of the GMP problem with the $s o(2,2)$ Laplace equation and the Schrödinger equation (3.16). It is easy to check that the change of variable and of function (2.4), (2.5), followed by the transformation

$$
y=\sinh ^{2} \theta \quad \Phi(y)=y^{-1 / 4}(1+y)^{-1 / 4} \Xi(y) \quad \Xi(y)=\chi(\theta)
$$

maps the Schrödinger equation for the GMP into that corresponding to the PTP, given in eq. (3.16). Therefore, we can use the results obtained for the latter to find the level spectrum and corresponding eigenfunctions of the GMP. For such purpose, we should identify

$$
\left|m_{1}\right|=2 \beta \quad\left|m_{2}\right|=2 \alpha \quad \bar{\epsilon}=4 C-1=-(2 l-1)^{2} .
$$

It then follows from eq. (3.17) that

$$
(2 l-1)^{2}=[2(\beta-\alpha)-1-2 n]^{2} .
$$


Hence, we directly obtain eq. (2.11), and from it the GMP spectrum (2.15).

Thus, we have shown that the GMP eigenvalue spectrum can be obtained by using the Laplace-Beltrami-Casimir operator for the so $(2,2)$ algebra. The eigenfunctions of the Laplace operator (or, equivalently, of the PTP Hamiltonian) are related to those of the GMP through eq. (3.19). We have therefore established that $s o(2,2)$ is the algebra responsible for the GMP exact solvability. In the next section, we shall proceed to construct its generators, and to study their action on the GMP wave functions.

\section{Symmetry algebra associated with the generalized Morse potential}

According to the analysis of the previous section, by interchanging the roles of energy and potential parameters, our original Schrödinger equation for the GMP can be mapped into the Schrödinger equation for the PTP (3.16), provided we make the identifications (3.20).

In terms of the variable $y$ defined in (3.19), the (unnormalized) solutions of the PTP Schrödinger equation can be written as

$$
\Xi(y)=y^{\alpha+1 / 4}(1+y)_{2}^{1 / 4-\beta} F_{1}(-n,-n+1-2 l ; 2 \alpha+1 ;-y)
$$

where use has been made of eqs. (2.5), (2.16), and (3.19). Let us define

$$
\left|m_{1}\right|=m-g=2 \beta \quad\left|m_{2}\right|=m+g=2 \alpha \quad \text { or } \quad m=\alpha+\beta \quad g=\alpha-\beta
$$

and make this substitution in eq. (4.1). Taking eq. (2.11) into account, we obtain

$$
\Xi_{m, g}^{(l)}(y)=y^{(g+m+1 / 2) / 2}(1+y)^{(g-m+1 / 2) / 2}{ }_{2} F_{1}(g+l, g+1-l ; m+g+1 ;-y)
$$

where for reasons that will soon become clear, we introduced upper and lower indices $(l)$, and $m, g$, respectively.

We are now able to show that the algebra so $(2,2)$ associated with the GMP can be explicitly represented as $s u(1,1) \oplus s u(1,1)$. In fact, according to Barut et al [5], for the

\footnotetext{
${ }^{1}$ The other solution of eq. (3.21) can easily be shown to violate the condition $\alpha>0$.
} 
PTP we can write down two sets of generators $\bar{G}^{+}, \bar{G}^{-}, \bar{G}_{3}$, and $\bar{M}^{+}, \bar{M}^{-}$, $\bar{M}_{3}$, each corresponding to an $s u(1,1)$ algebra, i.e., satisfying the relations

$$
\begin{aligned}
{\left[\bar{G}_{3}, \bar{G}_{ \pm}\right] } & = \pm \bar{G}_{ \pm} & {\left[\bar{G}_{+}, \bar{G}_{-}\right]=-2 \bar{G}_{3} } \\
{\left[\bar{M}_{3}, \bar{M}_{ \pm}\right] } & = \pm \bar{M}_{ \pm} & {\left[\bar{M}_{+}, \bar{M}_{-}\right]=-2 \bar{M}_{3} . }
\end{aligned}
$$

In terms of the variable $y$, and of two auxiliary variables $\xi, \eta \in[0,2 \pi)$, they are given by

$$
\begin{aligned}
\bar{G}^{ \pm}= & -\frac{1}{2} e^{ \pm i \eta}\left[\mp 2 \sqrt{y(1+y)} \frac{\partial}{\partial y}+\sqrt{\frac{1+y}{y}}\left(-i\left(\frac{\partial}{\partial \xi}+\frac{\partial}{\partial \eta}\right) \pm \frac{1}{2}\right)\right. \\
& \left.+\sqrt{\frac{y}{1+y}}\left(i\left(\frac{\partial}{\partial \xi}-\frac{\partial}{\partial \eta}\right) \pm \frac{1}{2}\right)\right] \\
\bar{G}_{3}= & -i \frac{\partial}{\partial \eta}
\end{aligned}
$$

and

$$
\begin{aligned}
& \bar{M}^{ \pm}=\frac{1}{2} e^{ \pm i \xi}\left[\mp 2 \sqrt{y(1+y)} \frac{\partial}{\partial y}+\sqrt{\frac{1+y}{y}}\left(-i\left(\frac{\partial}{\partial \xi}+\frac{\partial}{\partial \eta}\right) \pm \frac{1}{2}\right)\right. \\
& \left.+\sqrt{\frac{y}{1+y}}\left(-i\left(\frac{\partial}{\partial \xi}-\frac{\partial}{\partial \eta}\right) \pm \frac{1}{2}\right)\right] \\
& \bar{M}_{3}=-i \frac{\partial}{\partial \xi}
\end{aligned}
$$

respectively.

Applying them on the extended PTP wave functions

$$
\bar{\Xi}_{m, g}^{(l)}(\xi, y, \eta)=e^{i m \xi} \Xi_{m, g}^{(l)}(y) e^{i g \eta},
$$

we obtain after some calculations using well-known properties of the hypergeometric function 13

$$
\begin{aligned}
\bar{G}^{+} \bar{\Xi}_{m, g}^{(l)}(\xi, y, \eta) & =\frac{(l+g)(l-g-1)}{m+g+1} \bar{\Xi}_{m, g+1}^{(l)}(\xi, y, \eta) \\
\bar{G}^{-} \bar{\Xi}_{m, g}^{(l)}(\xi, y, \eta) & =-(m+g) \bar{\Xi}_{m, g-1}^{(l)}(\xi, y, \eta) \\
\bar{G}_{3} \bar{\Xi}_{m, g}^{(l)}(\xi, y, \eta) & =g \bar{\Xi}_{m, g}^{(l)}(\xi, y, \eta)
\end{aligned}
$$

and

$$
\begin{aligned}
\bar{M}^{+} \bar{\Xi}_{m, g}^{(l)}(\xi, y, \eta) & =\frac{(m+l)(m-l+1)}{m+g+1} \bar{\Xi}_{m+1, g}^{(l)}(\xi, y, \eta) \\
\bar{M}^{-} \bar{\Xi}_{m, g}^{(l)}(\xi, y, \eta) & =(m+g) \bar{\Xi}_{m-1, g}^{(l)}(\xi, y, \eta) \\
\bar{M}_{3} \bar{\Xi}_{m, g}^{(l)}(\xi, y, \eta) & =m \bar{\Xi}_{m, g}^{(l)}(\xi, y, \eta) .
\end{aligned}
$$


From eq. (3.19), the corresponding operators $G^{+}, G^{-}, G_{3}, M^{+}, M^{-}, M_{3}$ for the GMP can be written as $G^{+}=[y(1+y)]^{-1 / 4} \bar{G}^{+}[y(1+y)]^{1 / 4}$, and similarly for the other generators. Their detailed expressions are given by

$$
\begin{aligned}
G^{ \pm} & =-\frac{1}{2} e^{ \pm i \eta}\left[\mp 2 \sqrt{y(1+y)} \frac{\partial}{\partial y}-i \sqrt{\frac{1+y}{y}}\left(\frac{\partial}{\partial \xi}+\frac{\partial}{\partial \eta}\right)+i \sqrt{\frac{y}{1+y}}\left(\frac{\partial}{\partial \xi}-\frac{\partial}{\partial \eta}\right)\right] \\
G_{3} & =-i \frac{\partial}{\partial \eta} \\
M^{ \pm} & =\frac{1}{2} e^{ \pm i \xi}\left[\mp 2 \sqrt{y(1+y)} \frac{\partial}{\partial y}-i \sqrt{\frac{1+y}{y}}\left(\frac{\partial}{\partial \xi}+\frac{\partial}{\partial \eta}\right)-i \sqrt{\frac{y}{1+y}}\left(\frac{\partial}{\partial \xi}-\frac{\partial}{\partial \eta}\right)\right] \\
M_{3} & =-i \frac{\partial}{\partial \xi} .
\end{aligned}
$$

Defining now extended, normalized GMP wave functions by

$$
\bar{\Phi}_{m, g}^{(l)}(\xi, y, \eta)=(2 \pi)^{-1} e^{i m \xi} \Phi_{m, g}^{(l)}(y) e^{i g \eta}
$$

where $\Phi_{m, g}^{(l)}(y)$ is to be identified with the function $\Phi_{n}(y)$ obtained from eq. (2.16), we get

$$
\begin{aligned}
G^{+} \bar{\Phi}_{m, g}^{(l)}(\xi, y, \eta) & =-\left(\frac{(g+1)(m-g)(m+g)(g+l)(g-l+1)}{g(m-g-1)(m+g+1)}\right)^{1 / 2} \bar{\Phi}_{m, g+1}^{(l)}(\xi, y, \eta) \\
G^{-} \bar{\Phi}_{m, g}^{(l)}(\xi, y, \eta) & =-\left(\frac{(g-1)(m-g)(m+g)(g-l)(g+l-1)}{g(m-g+1)(m+g-1)}\right)^{1 / 2} \bar{\Phi}_{m, g-1}^{(l)}(\xi, y, \eta) \\
G_{3} \bar{\Phi}_{m, g}^{(l)}(\xi, y, \eta) & =g \bar{\Phi}_{m, g}^{(l)}(\xi, y, \eta) \\
M^{+} \bar{\Phi}_{m, g}^{(l)}(\xi, y, \eta) & =\left(\frac{(m-g)(m+g)(m+l)(m-l+1)}{(m-g+1)(m+g+1)}\right)^{1 / 2} \bar{\Phi}_{m+1, g}^{(l)}(\xi, y, \eta) \\
M^{-} \bar{\Phi}_{m, g}^{(l)}(\xi, y, \eta) & =\left(\frac{(m-g)(m+g)(m-l)(m+l-1)}{(m-g-1)(m+g-1)}\right)^{1 / 2} \bar{\Phi}_{m-1, g}^{(l)}(\xi, y, \eta) \\
M_{3} \bar{\Phi}_{m, g}^{(l)}(\xi, y, \eta) & =m \bar{\Phi}_{m, g}^{(l)}(\xi, y, \eta) .
\end{aligned}
$$

In deriving eqs. (4.12) and (4.13), we used the fact that the action of $G^{ \pm}, G_{3}, M^{ \pm}, M_{3}$ on the extended, unnormalized GMP wave functions is the same as that of $\bar{G}^{ \pm}, \bar{G}_{3}, \bar{M}^{ \pm}, \bar{M}_{3}$ on $\bar{\Xi}_{m, g}^{(l)}(\xi, y, \eta)$, as well as the expression (2.19) of the GMP wave function normalization coefficient.

We can also write down the expressions for the Casimir operators of each $s u(1,1)$ algebra,

$$
\mathcal{C}_{s u(1,1)_{I}}=-G^{+} G^{-}+G_{3}^{2}-G_{3} \quad \mathcal{C}_{s u(1,1)_{I I}}=-M^{+} M^{-}+M_{3}^{2}-M_{3} .
$$


From (4.12) and (4.13), it is easy to prove that their action on the extended wave functions 4.11 is

$$
\begin{aligned}
\mathcal{C}_{s u(1,1)_{I}} \bar{\Phi}_{m, g}^{(l)}(\xi, y, \eta) & =\mathcal{C}_{s u(1,1)_{I I}} \bar{\Phi}_{m, g}^{(l)}(\xi, y, \eta)=l(l-1) \bar{\Phi}_{m, g}^{(l)}(\xi, y, \eta) \\
& =-C \bar{\Phi}_{m, g}^{(l)}(\xi, y, \eta) .
\end{aligned}
$$

The irreducible representations of both $s u(1,1)$ algebras are therefore characterized by $l$ or, equivalently, by $C=-k b^{2}$, or by the combination $D b^{2} / a^{2}$ of the parameters regulating the depth, the position of the minimum, and the radius of the GMP, respectively (see eqs. (2.9) and (2.10)).

As we can see from eq. (4.12), the operators $G^{ \pm}$change $g$ into $g \pm 1$, respectively, without changing $m$. According to the definitions (4.2) of $g$ and $m$, and eqs. (2.11), (2.12),

$$
\begin{aligned}
g & =-l-n \quad n=0,1,2, \ldots \\
m & =\frac{C(b+2)}{g b}
\end{aligned}
$$

so that the action of $G^{ \pm}$forces $n$ and $b$ to change.

Let us analyse these changes in detail. Since $g \rightarrow g \pm 1$, and $C=-l(l-1)$ is a constant in a given irrep of $s u(1,1)_{I}$, we have that $n \rightarrow n \mp 1$. On the other hand, $m \rightarrow m$ means that $b$ should change from $b_{g}$ to

$$
b_{g \pm 1}=\frac{2 g b_{g}}{\left(2 g \pm b_{g} \pm 2\right)} .
$$

From the definition (2.9) of $C$, we then obtain $k_{g} \rightarrow k_{g \pm 1}, D_{g} \rightarrow D_{g \pm 1}, a_{g} \rightarrow a_{g \pm 1}$, where

$$
k_{g} b_{g}^{2}=k_{g \pm 1} b_{g \pm 1}^{2} \quad \frac{D_{g} b_{g}^{2}}{a_{g}^{2}}=\frac{D_{g \pm 1} b_{g \pm 1}^{2}}{a_{g \pm 1}^{2}} .
$$

As eq. (4.16) shows, the $s u(1,1)$ irreps associated with $g$ correspond to the negative discrete series. The highest-weight vector corresponds to $g=-l$ or $n=0$, and according to eq. (4.12), it satisfies the relation

$$
G^{+} \bar{\Phi}_{m,-l}^{(l)}(\xi, y, \eta)=0
$$

as it should be. Since the matrix representing $G^{+}$in the basis $\left\{\bar{\Phi}_{m, g}^{(l)}\right\}$ is not the adjoint of that representing $G^{-}$, the corresponding irrep is non-unitary. 
Let us now analyze the action of the operators $M^{ \pm}$, given in eq. (4.13). In this case $m \rightarrow m \pm 1$, and $g$ does not change. From eqs. (4.16) and (4.17), we see that $n$ does not change, and $b$ changes appropriately from $b_{m}$ to

$$
b_{m \pm 1}=\frac{2 C b_{m}}{2 C \pm g b_{m}} .
$$

From the constancy of $C$, we have again $k_{m} \rightarrow k_{m \pm 1}, D_{m} \rightarrow D_{m \pm 1}, a_{m} \rightarrow a_{m \pm 1}$, where

$$
k_{m} b_{m}^{2}=k_{m \pm 1} b_{m \pm 1}^{2} \quad \frac{D_{m} b_{m}^{2}}{a_{m}^{2}}=\frac{D_{m \pm 1} b_{m \pm 1}^{2}}{a_{m \pm 1}^{2}} .
$$

As it can be checked from eq. (4.13), the function $\bar{\Phi}_{l, g}^{(l)}$, corresponding to $m=l$, satisfies the relation

$$
M^{-} \bar{\Phi}_{l, g}^{(l)}(\xi, y, \eta)=0
$$

Hence, the $s u(1,1)$ irreducible representations associated with $m$ belong to the positive discrete series, i.e.,

$$
m=l+v \quad v=0,1,2, \ldots
$$

They are non-unitary, as already noted for those associated with $g$.

So as advertised in section 1, we have shown that the GMP so(2,2) symmetry algebra does not leave invariant the energy eigenvalue of a set of satellite potentials, but instead the parameter combination (1.6), where the function $f$ is given by $f=D b^{2} / a^{2}$, and for the index $m$ we may use either $g$ or $m$, as defined in eq. (4.2). As a final point, it is worth stressing that for the $s o(2,2)$ algebra (but not for the corresponding group $S O(2,2)$ ), the quantum number $l$, characterizing its irreducible representations, is not restricted to integer or half-integer values. Hence our algebraic formalism may be used for any real values of the GMP parameters $D, b$, and $a$, for which bound states do exist.

\section{Concluding remarks}

In the present paper, we did study in detail the bound state spectrum of the GMP, previously proposed by Deng and Fan [8] as a potential function for diatomic molecules. By connecting the corresponding Schrödinger equation with the Laplace equation on the hyperboloid and the Schrödinger equation for the PTP, we did explain the exact solvability of the 
problem by an $s o(2,2)$ symmetry algebra, giving rise to a set of satellite potentials of a new type. Such a symmetry algebra differs from the well-known potential algebras [2, 3, 4, 5] by the fact that its Casimir operators are not related to the Hamiltonian as for the latter, but to some function of the potential parameters.

It is worth noticing that some algebras with generators simultaneously changing the energy and the potential parameters, as the GMP so $(2,2)$ symmetry algebra, did already occur in another context. Some years ago, various attempts have indeed been made to combine features of both dynamical and potential algebras by enlarging the latter with some operators connecting eigenfunctions corresponding to the same potential parameters, but different energy eigenvalues [2, 5, 15]. The resulting algebras, referred to as dynamical potential algebras [15], may contain as substructures some algebras with the above-mentioned characteristics. However these subalgebras strikingly differ from the GMP symmetry algebra, in the sense that their Casimir operators are some complicated functions of both the Hamiltonian and the potential parameters, instead of the latter only.

The new type of satellite potentials introduced in the present paper may be physically relevant in the following context. The vibrational potentials $V(r)$ and $V^{\prime}(r)$, corresponding to different electronic states $K_{e}$ and $K_{e}^{\prime}$ of a diatomic molecule, are in general different. In an analysis of electromagnetic transitions between rovibrational bands, based on the electronic states $K_{e}$ and $K_{e}^{\prime}$ respectively, the corresponding eigenfunctions $\Psi(r)$ and $\Psi^{\prime}(r)$ in the potentials $V(r)$ and $V^{\prime}(r)$ should be used to calculate the Frank-Condon factors. The above-mentioned approach allows one to connect with one another the potentials $V(r)$ and $V^{\prime}(r)$, which might be taken as GMP's, by identifying them as members of the set of satellite potentials. The algebraic relation between the corresponding eigenfunctions $\Psi(r)$ and $\Psi^{\prime}(r)$, which were established here, would then significantly simplify the calculation of Frank-Condon factors. In such respect, the $s u(1,1)$ subalgebra of $s o(2,2)$ associated with $g$ looks more promising than that associated with $m$, since the operators $G^{ \pm}$, whose action is illustrated in fig. 2, could describe transitions where the vibrational molecular states (characterized by $n$ ), and the electronic states (belonging to definite satellite potentials) change simultaneously. We plan to analyse this point further in a forthcoming publication. 


\section{Acknowledgments}

Two of us (ADSM, YuFS) would like to thank Professor E Ley-Koo for his valuable suggestions and discussions on the subject. This work was supported in part by CONACYT, Mexico, by the Ministère de l'Education Nationale et de la Culture, Communauté Française

de Belgique, and by the Russian Foundation of the Fundamental Research, Grant No 9601-01421. 


\section{Appendix. SUSYQM analysis and normalization of wave functions}

The purpose of the present appendix is to briefly review the SUSYQM approach to the GMP problem, and to use it to prove eq. (2.19) for the wave function normalization coefficient.

Let us consider the Schrödinger equation (2.2) for the GMP in dimensionless variable $x$, and denote the corresponding Hamiltonian, potential, energies, and wave functions by $h^{0)}$, $v^{(0)}, \epsilon_{n}^{(0)}$, and $\psi_{n}^{(0)}(x)$, respectively. In SUSYQM [6, 7], the hamiltonian $h^{(0)}$ can be written in a factorized form, $h^{(0)}=A^{+} A^{-}+\epsilon_{0}^{(0)}$, in terms of the operators $A^{ \pm}=\mp d / d x+W(x)$, where the superpotential $W(x)$ is related to the ground state wave function $\psi_{0}^{(0)}(x)$ by $W(x)=-d\left(\ln \psi_{0}^{(0)}(x)\right) / d x$. By taking eq. (2.16) for $n=0$ into account, we get

$$
A^{ \pm}= \pm y(1+y) \frac{d}{d y}+\alpha_{0}+\left(\alpha_{0}-\beta_{0}\right) y=\mp \frac{d}{d x}+\frac{\alpha_{0} e^{x}-\beta_{0}}{e^{x}-1}
$$

The supersymmetric partner of $h^{(0)}$ is the Hamiltonian $h^{(1)}=A^{-} A^{+}+\epsilon_{0}^{(0)}$. From $h^{(0)}, h^{(1)}$, and $A^{ \pm}$, we can form the generators of an $s u(1 / 1)$ superalgebra, namely the supersymmetric Hamiltonian $\mathcal{H}$, and supercharges $Q^{ \pm}$, defined by

$$
\mathcal{H}=\left(\begin{array}{cc}
h^{(0)}-\epsilon_{0}^{(0)} & 0 \\
0 & h^{(1)}-\epsilon_{0}^{(0)}
\end{array}\right) \quad Q^{+}=\left(\begin{array}{cc}
0 & A^{+} \\
0 & 0
\end{array}\right) \quad Q^{-}=\left(\begin{array}{cc}
0 & 0 \\
A^{-} & 0
\end{array}\right)
$$

respectively, and satisfying the relations

$$
\left[\mathcal{H}, Q^{ \pm}\right]=0 \quad\left\{Q^{+}, Q^{-}\right\}=\mathcal{H} \quad\left\{Q^{ \pm}, Q^{ \pm}\right\}=0
$$

From eq. (A.1), we obtain

$$
h^{(1)}=-\frac{d^{2}}{d x^{2}}+v^{(1)}(x)
$$

where

$$
v^{(1)}(x)=v^{(0)}(x)+2 \frac{d W(x)}{d x}=k\left(1-\frac{b}{e^{x}-1}\right)^{2}+\frac{2 l e^{x}}{\left(e^{x}-1\right)^{2}}
$$

and $l$ is defined in eq. (2.10). The potential $v^{(1)}(x)$ can be rewritten as

$$
v^{(1)}(x)=k^{\prime}\left(1-\frac{b^{\prime}}{e^{x}-1}\right)^{2}+R\left(k^{\prime}, b^{\prime}\right)
$$


where

$$
k^{\prime}=\frac{(k b-l)^{2}}{k b^{2}+2 l} \quad b^{\prime}=\frac{k b^{2}+2 l}{k b-l} \quad R=k\left(k^{\prime}, b^{\prime}\right)-k^{\prime}=\frac{\left(k^{\prime} b^{\prime}-1+l^{\prime}\right)^{2}}{k^{\prime} b^{\prime 2}+2-2 l^{\prime}}-k^{\prime}
$$

and

$$
l^{\prime} \equiv \frac{1}{2}\left(1+\sqrt{1+4 k^{\prime} b^{\prime 2}}\right)=l+1
$$

hence showing that the GMP is a shape-invariant potential.

The eigenvalues $\epsilon_{n}^{(1)}$ of the supersymmetric partner are given by

$$
\epsilon_{n}^{(1)}=\epsilon_{n+1}^{(0)}=k-\alpha_{n+1}^{2}
$$

where in the last step, we used eq. (2.14). Due to the shape invariance, they can also be written as

$$
\epsilon_{n}^{(1)}=k^{\prime}-\alpha_{n}^{\prime 2}+R\left(k^{\prime}, b^{\prime}\right)=k-\alpha_{n}^{\prime 2}
$$

where $\alpha_{n}^{\prime}$ is defined in terms of $k^{\prime}, b^{\prime}, l^{\prime}$, and $n$ in the same way as $\alpha_{n}$ in terms of $k, b, l$, and $n$ (see eq. (2.13)). Comparing eq. (A.9) with eq. (A.10), and using eqs. (2.11), and (A.8), lead to the conclusion that

$$
\alpha_{n}^{\prime}=\alpha_{n+1} \quad \beta_{n}^{\prime}=\beta_{n+1} .
$$

Hence, the eigenfunctions of the supersymmetric partner are given by

$$
\psi_{n}^{(1)}=\frac{1}{\sqrt{a}} N_{n}^{\prime} y^{\alpha_{n+1}}(1+y)^{-\beta_{n+1}} F_{1}\left(-n,-n-1-2 l, 2 \alpha_{n+1}+1,-y\right)
$$

where $N_{n}^{\prime}$ can be obtained from $N_{n}$ by the substitutions $l \rightarrow l^{\prime}, \alpha_{n} \rightarrow \alpha_{n}^{\prime}, \beta_{n} \rightarrow \beta_{n}^{\prime}$.

From SUSYQM, however, we also know that $\psi_{n-1}^{(1)}$ can be obtained from the eigenfunction $\psi_{n}^{(0)}$ of $h^{(0)}$, corresponding to the same eigenvalue $\epsilon_{n}^{(0)}=\epsilon_{n-1}^{(1)}$, by applying the operator $A^{-}$:

$$
\psi_{n-1}^{(1)}=\left(\epsilon_{n}^{(0)}-\epsilon_{0}^{(0)}\right)^{-1 / 2} A^{-} \psi_{n}^{(0)}=\left(\alpha_{0}^{2}-\alpha_{n}^{2}\right)^{-1 / 2} A^{-} \psi_{n}^{(0)}
$$

With the help of eqs. (A.1), (2.16), and the relations

$$
\begin{aligned}
\alpha_{0}-\alpha_{n} & =\frac{n\left(2 \alpha_{n}+n+2 l\right)}{2 l} \\
\alpha_{0}-\beta_{0}-\alpha_{n}+\beta_{n} & =n
\end{aligned}
$$


deriving from (2.11) and (2.13), we obtain

$$
\begin{aligned}
A^{-} \psi_{n}^{(0)}= & \frac{1}{\sqrt{a}} N_{n} y^{\alpha_{n}}(1+y)^{-\beta_{n}}\left[-y(1+y) \frac{d}{d y}+\frac{n}{2 l}\left(2 \alpha_{n}+n+2 l\right)+n y\right] \\
& \times{ }_{2} F_{1}\left(-n,-n+1-2 l ; 2 \alpha_{n}+1 ;-y\right) .
\end{aligned}
$$

By successively using the second relation in eq. (4.9.7) of ref. [1], and eq. (1.4.2) of ref. [13], eq. (A.16) can be transformed into

$$
\begin{aligned}
A^{-} \psi_{n}^{(0)} & \\
= & \frac{1}{\sqrt{a}} \frac{2 \alpha_{n}+n+2 l}{2 l} N_{n} y^{\alpha_{n}}(1+y)^{-\beta_{n}}\left[(n+2 l)_{2} F_{1}\left(-n,-n+1-2 l ; 2 \alpha_{n}+1 ;-y\right)\right. \\
& \left.-2 l_{2} F_{1}\left(-n,-n-2 l ; 2 \alpha_{n}+1 ;-y\right)\right] \\
= & \frac{1}{\sqrt{a}} \frac{n\left(2 \alpha_{n}+n+2 l\right)}{2 l} N_{n} y^{\alpha_{n}}(1+y)^{-\beta_{n}} F_{1}\left(-n+1,-n-2 l ; 2 \alpha_{n}+1 ;-y\right) .
\end{aligned}
$$

Finally, by combining eqs. A.13), (A.14), (A.17) with the relation

$$
\alpha_{0}+\alpha_{n}=2 \alpha_{n}+\left(\alpha_{0}-\alpha_{n}\right)=\frac{(n+2 l)\left(2 \alpha_{n}+n\right)}{2 l}
$$

we get

$$
\begin{aligned}
\psi_{n-1}^{(1)}= & {\left[\frac{n\left(2 \alpha_{n}+n+2 l\right)}{a(n+2 l)\left(2 \alpha_{n}+n\right)}\right]^{1 / 2} N_{n} y^{\alpha_{n}}(1+y)^{-\beta_{n}} } \\
& \times{ }_{2} F_{1}\left(-n+1,-n-2 l ; 2 \alpha_{n}+1 ;-y\right) .
\end{aligned}
$$

We have therefore derived two equivalent expressions (A.12), and (A.19) for the wave functions of the GMP supersymmetric partner. By equating them, we obtain the following recursion relation for the normalization coefficients

$$
N_{n}=\left[\frac{(n+2 l)\left(2 \alpha_{n}+n\right)}{n\left(2 \alpha_{n}+n+2 l\right)}\right]^{1 / 2} N_{n-1}^{\prime} .
$$

The recursion starting value is the ground state normalization coefficient, which can be expressed in terms of beta or gamma functions, as follows:

$$
\begin{aligned}
N_{0} & =\left[a^{-1} \int_{0}^{\infty} d y y^{2 \alpha_{0}-1}(1+y)^{-2 \beta_{0}-1}\right]^{-1 / 2}=\left[a^{-1} B\left(2 \alpha_{0}, 2 \beta_{0}-2 \alpha_{0}+1\right)\right]^{-1 / 2} \\
& =\left[\frac{a \Gamma\left(2 \beta_{0}+1\right)}{\Gamma\left(2 \alpha_{0}\right) \Gamma\left(2 \beta_{0}-2 \alpha_{0}+1\right)}\right]^{1 / 2}=\left[\frac{a \Gamma\left(2 \alpha_{0}+2 l+1\right)}{\Gamma\left(2 \alpha_{0}\right) \Gamma(2 l+1)}\right]^{1 / 2}
\end{aligned}
$$

It is then a simple matter to prove by using eqs. (A.8), and (A.11), that eq. (2.19) provides the solution of eqs. (A.20), and (A.21). 


\section{References}

[1] Infeld L and Hull TE 1951 Rev. Mod. Phys. 2321

[2] Alhassid Y, Gürsey F and Iachello F 1983 Ann. Phys., NY 148346

[3] Alhassid Y, Gürsey F and Iachello F 1986 Ann. Phys., NY 167181

Wu J, Alhassid Y and Gürsey F 1989 Ann. Phys., NY 196163

Wu J and Alhassid Y 1990 J. Math. Phys. 31557

[4] Frank A and Wolf K B 1984 Phys. Rev. Lett. 52 1737; 1985 J. Math. Phys. 26973

[5] Barut A O, Inomata A and Wilson R 1987 J. Phys. A: Math. Gen. 20 4075, 4083

[6] Gendenshtein L E 1983 Zh. Eksp. Theor. Fiz. Pis. Red. 38299 (JETP Lett. 38 356)

[7] Dabrowska J W, Khare A and Sukhatme U P 1988 J. Phys. A: Math. Gen. 21 L195 Cooper F, Khare A and Sukhatme U 1995 Phys. Rep. 251267

[8] Deng Z H and Fan Y P 1957 Shandong University Journal 7162 (in Chinese)

[9] Morse P M 1929 Phys. Rev. 3457

[10] Nicholls R W 1982 J. Quantum Spectrosc. Radiat. Trans. 28481

Palma A and Morales J 1983 Int. J. Quantum Chem. S17 393

Morales J, Palma A and Berrondo M 1984 Int. J. Quantum Chem. S18 57

Drallos P J and Wadhera J M 1986 J. Chem. Phys. 856524

Ley-Koo E, Mateos S and Villa-Torres G 1995 Int. J. Quantum Chem. 56175

[11] Smirnov Yu F and Shustov A P 1981 Yad. Phys. 34626 (Sov. J. Nucl. Phys. 34 349)

[12] Manning M F and Rosen N 1933 Phys. Rev. 44953

[13] Slater L J 1966 Generalized Hypergeometric Functions (Cambridge: Cambridge UP)

[14] Ráczka R, Limić N and Niederle J 1966 J. Math. Phys. 71861 
[15] Quesne C 1988 J. Phys. A: Math. Gen. 21 4487, 4501; 1989 J. Phys. A: Math. Gen. 223723

Englefield M J and Quesne C 1991 J. Phys. A: Math. Gen. 243557 


\section{Figure captions}

Figure 1. Comparison between the GMP (full line), and the Morse and harmonic oscillator potentials (broken and chain lines respectively). The parameters of the first two are $a=1$, $r_{e}$ or $x_{e}=2.5, D=10$, while the frequency of the latter is $\hbar \omega=\epsilon(1)$.

Figure 2. Effects of the operators $G^{ \pm}$on the eigenfunctions of the GMP $(a=1)$. In each case, only the first three energy levels are shown. 